\title{
On some Opial-type inequalities
}

\author{
Chang-Jian Zhao ${ }^{1^{*}}$ and Wing-Sum Cheung ${ }^{2}$
}

* Correspondence: chjzhao@163. com

${ }^{1}$ Department of Mathematics, China Jiliang University, Hangzhou 310018, PR China

Full list of author information is available at the end of the article

\section{Abstract}

In the present paper we establish some new Opial-type inequalities involving higherorder partial derivatives. Our results in special cases yield some of the recent results on Opial's inequality and also provide new estimates on inequalities of this type.

MR (2000) Subject Classification 26D15

Keywords: Opial?'?s inequality, Opial-type integral inequalities, H?ö?lder?'?s inequality

\section{Introduction}

In the year 1960, Opial [1] established the following integral inequality:

Theorem 1.1. Suppose $f \in C^{1}[0, h]$ satisfies $f(0)=f(h)=0$ and $f(x)>0$ for all $x \in(0$, h). Then the integral inequality holds

$$
\int_{0}^{h}\left|f(x) f^{\prime}(x)\right| \mathrm{d} x \leq \frac{h}{4} \int_{0}^{h}\left(f^{\prime}(x)\right)^{2} \mathrm{~d} x
$$

where this constant $\frac{h}{4}$ is best possible.

Opial's inequality and its generalizations, extensions and discretizations play a fundamental role in establishing the existence and uniqueness of initial and boundary value problems for ordinary and partial differential equations as well as difference equations [2-6]. The inequality (1.1) has received considerable attention, and a large number of papers dealing with new proofs, extensions, generalizations, variants and discrete analogues of Opial's inequality have appeared in the literature [7-22]. For an extensive survey on these inequalities, see [2,6]. For Opial-type integral inequalities involving high-order partial derivatives see [23-27]. The main purpose of the present paper is to establish some new Opial-type inequalities involving higher-order partial derivatives by an extension of Das's idea [28]. Our results in special cases yield some of the recent results on Opial's-type inequalities and provide some new estimates on such types of inequalities.

\section{Main results}

Let $n \geq 1, k \geq 1$. Our main results are given in the following theorems.

Theorem 2.1 Let $x(s, t) \in C^{(n-1)}[0, a] \times C^{(k-1)}[0, b]$ be such that $\frac{\partial^{i}}{\partial \sigma^{i}} x(0, \tau)=0$, $\frac{\partial^{j}}{\partial \tau^{j}} x(\sigma, 0)=0, \sigma \in[0, s], \tau \in[0, t], 0 \leq i \leq n-1,0 \leq j \leq k-1$. Further, let $\frac{\partial^{n-1}}{\partial s^{n-1}} x(s, t)$, $\frac{\partial^{k-1}}{\partial t^{k-1}} x(s, t)$ be absolutely continuous, and $\int_{0}^{a} \int_{0}^{b}\left|x^{(n, k)}(s, t)\right|^{2} \mathrm{~d} s \mathrm{~d} t<\infty$. Then

\section{SpringerOpen ${ }^{\circ}$}

(c) 2011 Zhao and Cheung; licensee Springer. This is an Open Access article distributed under the terms of the Creative Commons Attribution License (http://creativecommons.org/licenses/by/2.0), which permits unrestricted use, distribution, and reproduction in any medium, provided the original work is properly cited. 


$$
\int_{0}^{a} \int_{0}^{b}\left|x(s, t) \cdot x^{(n, k)}(s, t)\right| \mathrm{d} s \mathrm{~d} t \leq c_{n, k} \cdot a^{n} b^{k} \cdot \int_{0}^{a} \int_{0}^{b}\left|x^{(n, k)}(s, t)\right|^{2} \mathrm{~d} s \mathrm{~d} t
$$

where

$$
x^{(n, k)}(s, t)=\frac{\partial^{n}}{\partial s^{n}}\left(\frac{\partial^{k}}{\partial t^{k}} x(s, t)\right),
$$

and

$$
c_{n, k}=\frac{1}{4 n ! k !}\left(\frac{2 n k}{(2 n-1)(2 k-1)}\right)^{\frac{1}{2}} .
$$

Proof. For $\sigma$ integration by parts $(n-1)$-times and in view of $\frac{\partial^{i}}{\partial \sigma^{i}} x(0, \tau)=0$, $\frac{\partial^{j}}{\partial \tau^{j}} x(\sigma, 0)=0,0 \leq i \leq n-1,0 \leq j \leq k-1$ we have

$$
\begin{gathered}
x(s, t)=\frac{(-1)^{n}}{(n-1) !} \int_{s}^{0}(\sigma-s)^{n-1} \frac{\partial^{n}}{\partial \sigma^{n}} x(\sigma, t) \mathrm{d} \sigma \\
=\frac{(-1)^{2 n-1}}{(n-1) !} \int_{s}^{0}(s-\sigma)^{n-1} \frac{\partial^{n}}{\partial \sigma^{n}} x(\sigma, t) \mathrm{d} \sigma=\frac{1}{(n-1) !} \int_{0}^{s}(s-\sigma)^{n-1} \frac{\partial^{n}}{\partial \sigma^{n}} x(\sigma, t) \mathrm{d} \sigma \\
=\frac{1}{(n-1) !(k-1) !} \int_{0}^{s}(s-\sigma)^{n-1} \frac{\partial^{n}}{\partial \sigma^{n}}\left(\int_{0}^{t}(t-\tau)^{k-1} \frac{\partial^{k}}{\partial \tau^{k}} x(\sigma, \tau) d \tau\right) \mathrm{d} \sigma \\
=\frac{1}{(n-1) !(k-1) !} \int_{0}^{s} \int_{0}^{t}(s-\sigma)^{n-1}(t-\tau)^{k-1} \cdot x^{(n, k)}(\sigma, \tau) \mathrm{d} \sigma \mathrm{d} \tau .
\end{gathered}
$$

Multiplying both sides of (2.2) by $x^{(n, k)}(s, t)$ and using the Cauchy-Schwarz inequality, we have

$$
\begin{gathered}
\left|x(s, t) \cdot x^{(n, k)}(s, t)\right| \leq \frac{\left|x^{(n, k)}(s, t)\right|}{(n-1) !(k-1) !}\left(\int_{0}^{s} \int_{0}^{t}(s-\sigma)^{2 n-2}(t-\tau)^{2 k-2} \mathrm{~d} \sigma \mathrm{d} \tau\right)^{\frac{1}{2}} \\
\times\left(\int_{0}^{s} \int_{0}^{t}\left|x^{(n, k)}(\sigma, \tau)\right|^{2} \mathrm{~d} \sigma \mathrm{d} \tau\right)^{\frac{1}{2}} \\
=\frac{1}{(n-1) !(k-1) ! \sqrt{(2 n-1)(2 k-1)}} \cdot s^{n-\frac{1}{2}} t^{k-\frac{1}{2}}\left|x^{(n, k)}(s, t)\right|\left(\int_{0}^{s} \int_{0}^{t}\left|x^{(n, k)}(\sigma, \tau)\right|^{2} \mathrm{~d} \sigma \mathrm{d} \tau\right)^{\frac{1}{2}} .
\end{gathered}
$$

Thus, integrating both sides of (2.3) over $t$ from 0 to $b$ first and then integrating the resulting inequality over $s$ from 0 to $a$ and applying the Cauchy-Schwarz inequality again, we obtain

$$
\begin{gathered}
\int_{0}^{a} \int_{0}^{b}\left|x(s, t) \cdot x^{(n, k)}(s, t)\right| \mathrm{d} s \mathrm{~d} t \\
\leq \frac{1}{(n-1) !(k-1) ! \sqrt{(2 n-1)(2 k-1)}}\left(\int_{0}^{a} \int_{0}^{b} s^{2 n-1} t^{2 k-1} \mathrm{~d} s \mathrm{~d} t\right)^{\frac{1}{2}} \\
\times\left(\int_{0}^{a} \int_{0}^{b}\left|x^{(n, k)}(s, t)\right|^{2}\left(\int_{0}^{s} \int_{0}^{t}\left|x^{(n, k)}(\sigma, \tau)\right|^{2} \mathrm{~d} \sigma \mathrm{d} \tau\right) \mathrm{d} s \mathrm{~d} t\right)^{\frac{1}{2}} \\
=\frac{1}{2 n !}\left(\frac{2 n}{2 n-1}\right)^{\frac{1}{2}} \frac{1}{2 k !}\left(\frac{2 k}{2 k-1}\right)^{\frac{1}{2}} a^{n} b^{k} \\
\times\left(\frac{1}{2} \int_{0}^{a} \int_{0}^{b} \frac{\partial^{2}}{\partial s \partial t}\left\{\left(\int_{0}^{s} \int_{0}^{t}\left|x^{(n, k)}(\sigma, \tau)\right|^{2} \mathrm{~d} \sigma \mathrm{d} \tau\right)^{2}\right\} \mathrm{d} s \mathrm{~d} t\right)^{\frac{1}{2}} \\
=c_{n, k} a^{n} b^{k} \int_{0}^{a} \int_{0}^{b}\left|x^{(n, k)}(s, t)\right|^{2} \mathrm{~d} s \mathrm{~d} t .
\end{gathered}
$$


This completes the proof.

Remark 2.1. Let $x(s, t)$ reduce to $s(t)$ and with suitable modifications, Then (2.1) becomes the following inequality:

$$
\int_{0}^{a}\left|x(t) x^{(n)}(t)\right| \mathrm{d} t \leq \frac{1}{2 n !} \cdot\left(\frac{n}{2 n-1}\right)^{\frac{1}{2}} a^{n} \int_{0}^{a}\left|x^{(n)}(t)\right|^{2} \mathrm{~d} t .
$$

This is just an inequality established by Das [28]. Obviously, for $n \geq 2,(2.4)$ is sharper than the following inequality established by Willett [29].

$$
\int_{0}^{a}\left|x(t) x^{n}(t)\right| \mathrm{d} t \leq \frac{1}{2} a^{n} \int_{0}^{a}\left|x^{n}(t)\right|^{2} \mathrm{~d} t .
$$

Remark 2.2. Taking for $n=k=1$ in (2.1), (2.1) reduces to

$$
\int_{0}^{a} \int_{0}^{b}\left|x(s, t) \cdot \frac{\partial^{2}}{\partial s \partial t} x(s, t)\right| \mathrm{d} s \mathrm{~d} t \leq \frac{\sqrt{2}}{4} a b \int_{0}^{a} \int_{0}^{b}\left|\frac{\partial^{2}}{\partial s \partial t} x(s, t)\right|^{2} \mathrm{~d} s \mathrm{~d} t .
$$

Let $x(s, t)$ reduce to $s(t)$ and with suitable modifications. Then (2.6) becomes the following inequality: If $x(t)$ is absolutely continuous in $[0, a]$ and $x(0)=0$, then

$$
\int_{0}^{a}\left|x(t) x^{\prime}(t)\right| \mathrm{d} t \leq \frac{a}{2} \int_{0}^{a}\left|x^{\prime}(t)\right|^{2} \mathrm{~d} t .
$$

This is just an inequality established by Beesack [30].

Remark 2.3. Let $0 \leq \alpha, \beta<n$, but fixed, and let $g(s, t) \in C^{(n-\alpha-1)}[0, a] \times C^{(k-\beta-1)}[0, b]$ be such that $\frac{\partial^{i}}{\partial s^{i}} g(0, t)=\frac{\partial^{i}}{\partial t^{i}} g(s, 0)=0,0 \leq i \leq n-\alpha-1,0 \leq i \leq k-\beta-1$ and suppose that $\frac{\partial^{n-\alpha-1}}{\partial s^{n-\alpha-1}} g(s, t), \quad \frac{\partial^{k-\beta-1}}{\partial t^{k-\beta-1}} g(s, t) \quad$ are absolutely continuous, and $\int_{0}^{a} \int_{0}^{b}\left|x^{(n-\alpha, k-\beta)}(s, t)\right|^{2} \mathrm{~d} s \mathrm{~d} t<\infty$.

Then from (2.1) it follows that

$$
\int_{0}^{a} \int_{0}^{b}\left|g(s, t) \cdot g^{(n-\alpha, k-\beta)}(s, t)\right| \mathrm{d} s \mathrm{~d} t \leq c_{n-\alpha, k-\beta} a^{n-\alpha} b^{k-\beta} \int_{0}^{a} \int_{0}^{b}\left|g^{(n-\alpha, k-\beta)}(s, t)\right|^{2} \mathrm{~d} s \mathrm{~d} t .
$$

Thus, for $g(s, t)=x^{(\alpha, \beta)}(s, t)$, where $x(s, t) \in C^{(n-1)}[0, a] \times C^{(k-1)}[0, b], \frac{\partial^{i}}{\partial s^{i}} x(0, t)=0$, $\frac{\partial^{j}}{\partial t^{t}} x(s, 0)=0, \alpha \leq i \leq n-1, \beta \leq j \leq k-1$, and $x^{(n-1, \mathrm{k}-1)}(s, t)$ are absolutely continuous, and $\int_{0}^{a} \int_{0}^{b}\left|x^{(n, k)}(s, t)\right|^{2} \mathrm{~d} s \mathrm{~d} t<\infty$, then

$$
\int_{0}^{a} \int_{0}^{b}\left|x^{(\alpha, \beta)}(s, t) \cdot x^{(n, k)}(s, t)\right| \mathrm{d} s \mathrm{~d} t \leq c_{n-\alpha, k-\beta} a^{n-\alpha} b^{k-\beta} \int_{0}^{a} \int_{0}^{b}\left|x^{(n, k)}(s, t)\right|^{2} \mathrm{~d} s \mathrm{~d} t .
$$

Obviously, a special case of (2.7) is the following inequality:

$$
\int_{0}^{a} \int_{0}^{b}\left|x^{(k, k)}(s, t) \cdot x^{(n, n)}(s, t)\right| \mathrm{d} s \mathrm{~d} t \leq c_{n-k, n-k}(a b)^{n-k} \int_{0}^{a} \int_{0}^{b}\left|x^{(n, n)}(s, t)\right|^{2} \mathrm{~d} s \mathrm{~d} t(2.8)
$$

Let $x(s, t)$ reduce to $s(t)$ and with suitable modifications. Then (2.8) becomes the following inequality: 


$$
\int_{0}^{a}\left|x^{(k)}(t) x^{(n)}(t)\right| \mathrm{d} t \leq \frac{1}{2(n-k) !} \cdot\left(\frac{n-k}{2(n-k)-1}\right)^{\frac{1}{2}} a^{n-k} \int_{0}^{a}\left|x^{(n)}(t)\right|^{2} \mathrm{~d} t .
$$

This is just an inequality established by Agarwal and Thandapani [31].

Theorem 2.2. Let $l$ and $m$ be positive numbers satisfying $l+m>1$. Further, let $x(s, t)$ $\in C^{(n-1)}[0, a] \times C^{(k-1)}[0, b]$ be such that $\frac{\partial^{i}}{\partial \sigma^{i}} x(0, \tau)=0, \frac{\partial^{j}}{\partial \tau^{j}} x(\sigma, 0)=0, \sigma \in[0, s], \tau \in$ $[0, t], 0 \leq i \leq n-1,0 \leq j \leq k-1$ and assume that $\frac{\partial^{n-1}}{\partial s^{n-1}} x(s, t), \frac{\partial^{k-1}}{\partial t^{k-1}} x(s, t)$ are absolutely continuous, and $\int_{0}^{a} \int_{0}^{b}\left|x^{(n, k)}(s, t)\right|^{l+m} \mathrm{~d} s \mathrm{~d} t<\infty$. Then

$$
\int_{0}^{a} \int_{0}^{b}|x(s, t)|^{l}\left|x^{(n, k)}(s, t)\right|^{m} \mathrm{~d} s \mathrm{~d} t \leq c_{n, k}^{*} a^{n l} b^{k l} \int_{0}^{a} \int_{0}^{b}\left|x^{(n, k)}(s, t)\right|^{l+m} \mathrm{~d} s \mathrm{~d} t,
$$

where

$$
c_{n, k}^{*}=\xi^{l \xi+1} m^{\xi m}\left(\frac{k n(1-\xi)^{2}}{(n-\xi)(k-\xi)}\right)^{l(1-\xi)} \cdot(n ! k !)^{-l}, \quad \xi=\frac{1}{l+m} .
$$

Proof. From (2.2), we have

$$
|x(s, t)| \leq \frac{1}{(n-1) !(k-1) !} \int_{0}^{s} \int_{0}^{t}(s-\sigma)^{n-1}(t-\tau)^{k-1}\left|x^{(n, k)}(\sigma, \tau)\right| \mathrm{d} \sigma \mathrm{d} \tau,
$$

by Hölder's inequality with indices $l+m$ and $\frac{l+m}{l+m-1}$, it follows that

$$
\begin{aligned}
|x(s, t)| \leq \frac{1}{(n-1) !(k-1) !}\left(\int_{0}^{s} \int_{0}^{t}\left[(s-\sigma)^{n-1}(t-\tau)^{k-1}\right]^{\frac{l+m}{l+m-1}} \mathrm{~d} \sigma \mathrm{d} \tau\right)^{\frac{l+m-1}{l+m}} \\
\times\left(\int_{0}^{s} \int_{0}^{t}\left|x^{(n, k)}(\sigma, \tau)\right|^{l+m} \mathrm{~d} \sigma \mathrm{d} \tau\right)^{\frac{1}{l+m}} \\
=A s^{n-\xi} t^{k-\xi}\left(\int_{0}^{s} \int_{0}^{t}\left|x^{(n, k)}(\sigma, \tau)\right|^{l+m} \mathrm{~d} \sigma \mathrm{d} \tau\right)^{\xi}
\end{aligned}
$$

where

$$
A=\left(\frac{(1-\xi)^{2}}{(n-\xi)(k-\xi)}\right)^{1-\xi} \frac{1}{(n-1) !(k-1) !} .
$$

Multiplying the both sides of above inequality by $\left|x^{(n, k)}(s, t)\right|^{m}$ and integrating both sides over $t$ from 0 to $b$ first and then integrating the resulting inequality over $s$ from 0 to $a$, we obtain

$$
\begin{gathered}
\int_{0}^{a} \int_{0}^{b}|x(s, t)|^{l}\left|x^{(n, k)}(s, t)\right|^{m} \mathrm{~d} s \mathrm{~d} t \\
\leq A^{l} \int_{0}^{a} \int_{0}^{b} s^{l(n-\xi)} t^{l(k-\xi)}\left|x^{(n, k)}(s, t)\right|^{m}\left(\int_{0}^{s} \int_{0}^{t}\left|x^{(n, k)}(\sigma, \tau)\right|^{l+m} \mathrm{~d} \sigma \mathrm{d} \tau\right)^{l \xi} \mathrm{d} s \mathrm{~d} t .
\end{gathered}
$$


Now, applying Hölder's inequality with indices $\frac{l+m}{l}$ and $\frac{l+m}{m}$ to the integral on the right-side, we obtain

$$
\begin{gathered}
\int_{0}^{a} \int_{0}^{b}|x(s, t)|^{l}\left|x^{(n, k)}(s, t)\right|^{m} \mathrm{~d} s \mathrm{~d} t \leq A^{l}\left(\int_{0}^{a} \int_{0}^{b} s^{(n-\xi)(l+m)} t^{(k-\xi)(l+m)} \mathrm{d} s \mathrm{~d} t\right)^{\frac{l}{l+m}} \\
\times\left(\int_{0}^{a} \int_{0}^{b}\left|x^{(n, k)}(s, t)\right|^{m+l}\left(\int_{0}^{s} \int_{0}^{t}\left|x^{(n, k)}(\sigma, \tau)\right|^{l+m} \mathrm{~d} \sigma \mathrm{d} \tau\right)^{\frac{l}{m}} \mathrm{~d} s \mathrm{~d} t\right)^{\frac{l}{l+m}} \\
=A^{l}\left(\int_{0}^{a} \int_{0}^{b} s^{(n-\xi)(l+m)} t^{(k-\xi)(l+m)} \mathrm{d} s \mathrm{~d} t\right)^{\frac{l}{l+m}} \frac{m}{l+m} \\
\left.\int_{0}^{a} \int_{0}^{b} \frac{\partial^{2}}{\partial s \partial t}\left(\int_{0}^{s} \int_{0}^{t}\left|x^{(n, k)}(\sigma, \tau)\right|^{l+m} \mathrm{~d} \sigma \mathrm{d} \tau\right)^{\frac{l}{m}+1} \mathrm{~d} s \mathrm{~d} t\right)^{\frac{m}{l+m}} \\
=A^{l}\left(\frac{\xi^{2}}{k n}\right)^{\xi l}(m \xi)^{m \xi} a^{n l} b^{k l} \int_{0}^{a} \int_{0}^{b}\left|x^{(n, k)}(s, t)\right|^{l+m} \mathrm{~d} s \mathrm{~d} t \\
=c_{n, k}^{*} a^{n l} b^{k l} \int_{0}^{a} \int_{0}^{b}\left|x^{(n, k)}(s, t)\right|^{l+m} \mathrm{~d} s \mathrm{~d} t .
\end{gathered}
$$

This completes the proof.

Remark 2.4. Let $x(s, t)$ reduce to $s(t)$ and with suitable modifications. Then (2.9) becomes the following inequality:

$$
\int_{0}^{a}|x(t)|^{l}\left|x^{(n)}(t)\right|^{m} \mathrm{~d} t \leq \xi m^{m \xi}\left(\frac{n(1-\xi)}{n-\xi}\right)^{l(1-\xi)}(n !)^{-l} a^{n l} \int_{0}^{a}\left|x^{(n)}(t)\right|^{l+m} \mathrm{~d} t .
$$

This is an inequality given by Das [28]. Taking for $n=1$ in (2.10), we have

$$
\int_{0}^{a}|x(t)|^{l}\left|x^{\prime}(t)\right|^{m} \mathrm{~d} t \leq \frac{m^{m /(l+m)}}{l+m} a^{l} \int_{0}^{a}\left|x^{\prime}(t)\right|^{m+l} \mathrm{~d} t .
$$

For $m, l \geq 1$ Yang [32] established the following inequality:

$$
\int_{0}^{a}|x(t)|^{l}\left|x^{\prime}(t)\right|^{m} \mathrm{~d} t \leq \frac{m}{l+m} a^{l} \int_{0}^{a}\left|x^{\prime}(t)\right|^{m+l} \mathrm{~d} t
$$

Obviously, for $m, l \geq 1$, (2.11) is sharper than (2.12).

Remark 2.5. For $n=k=1$; (2.9) reduces to

$$
\int_{0}^{a} \int_{0}^{b}|x(s, t)|^{l}\left|\frac{\partial^{2}}{\partial s \partial t} x(s, t)\right|^{m} \mathrm{~d} s \mathrm{~d} t \leq c_{1,1}^{*}(a b)^{l} \int_{0}^{a} \int_{0}^{b}\left|\frac{\partial^{2}}{\partial s \partial t} x(s, t)\right|^{m+l} \mathrm{~d} s \mathrm{~d} t .
$$

Let $x(s, t)$ reduce to $s(t)$ and with suitable modifications. Then above inequality becomes the following inequality:

$$
\int_{0}^{a}|x(t)|^{l}\left|x^{\prime}(t)\right|^{m} \mathrm{~d} t \leq \xi m^{m \xi} a^{l} \int_{0}^{a}\left|x^{\prime}(t)\right|^{m+l} \mathrm{~d} t, \quad \xi=(l+m)^{-1} .
$$


This is just an inequality established by Yang [32].

Remark 2.6. Following Remark 2.3, for $x(s, t) \in C^{(n-1)}[0, a] \times C^{(k-1)}[0, b]$, $\frac{\partial^{j}}{\partial t^{j}} x(s, 0)=0, \frac{\partial^{j}}{\partial t^{j}} x(s, 0)=0, \alpha \leq i \leq n-1, \beta \leq j \leq k-1$ and $x^{(n-1, k-1)}(s, t)$ are absolutely continuous, and $\int_{0}^{a} \int_{0}^{b}\left|x^{(n, k)}(s, t)\right|^{l+m} \mathrm{~d} s \mathrm{~d} t<\infty$, it is easy to obtain that

$$
\int_{0}^{a} \int_{0}^{b}\left|x^{(\alpha, \beta)}(s, t)\right|^{l} \cdot\left|x^{(n, k)}(s, t)\right|^{m} \mathrm{~d} s \mathrm{~d} t \leq c_{n-\alpha, k-\beta}^{*} a^{l(n-\alpha)} b^{l(k-\beta)} \int_{0}^{a} \int_{0}^{b}\left|x^{(n, k)}(s, t)\right|^{l+m} \mathrm{~d} s \mathrm{~d} t(2 .
$$

Obviously, a special case of (2.14) is the following inequality:

$$
\int_{0}^{a} \int_{0}^{b}\left|x^{(k, k)}(s, t)\right|^{l} \cdot\left|x^{(n, n)}(s, t)\right|^{m} \mathrm{~d} s \mathrm{~d} t \leq c_{n-k, n-k}^{*}(a b)^{l(n-k)} \int_{0}^{a} \int_{0}^{b}\left|x^{(n, n)}(s, t)\right|^{l+m} \mathrm{~d} s \mathrm{~d} t(2.14)
$$

Let $x(s, t)$ reduce to $s(t)$ and with suitable modifications, then (2.14) becomes the following inequality:

$$
\begin{gathered}
\int_{0}^{a}\left|x^{(k)}(t)\right|^{l}\left|x^{(n)}(t)\right|^{m} \mathrm{~d} t \\
\leq \xi m^{m \xi}\left(\frac{(n-k)(1-\xi)}{n-k-\xi}\right)^{l(1-\xi)}((n-k) !)^{-l} a^{(n-k) l} \int_{0}^{a}\left|x^{(n)}(t)\right|^{l+m} \mathrm{~d} t, \quad \xi=(l+m)^{-1} .
\end{gathered}
$$

This is just an inequality established by Agarwal and Thandapani [31].

Theorem 2.3. Let $l$ and $m$ be positive numbers satisfying $l+m=1$. Further, let $x(s$, $t) \in C^{(n-1)}[0, a] \times C^{(k-1)}[0, b]$ be such that $\frac{\partial^{i}}{\partial \sigma^{i}} x(0, \tau)=0, \frac{\partial^{j}}{\partial \tau^{j}} x(\sigma, 0)=0, \sigma \in[0, s]$, $\tau \in[0, t], 0 \leq i \leq n-1,0 \leq j \leq k-1$ and assume that $\frac{\partial^{n-1}}{\partial s^{n-1}} x(s, t), \frac{\partial^{k-1}}{\partial t^{k-1}} x(s, t)$ are absolutely continuous, and $\int_{0}^{a} \int_{0}^{b}\left|x^{(n, k)}(s, t)\right| \mathrm{d} s \mathrm{~d} t<\infty$. Then

$$
\int_{0}^{a} \int_{0}^{b}|x(s, t)|^{l}\left|x^{(n, k)}(s, t)\right|^{m} \mathrm{~d} s \mathrm{~d} t \leq \frac{m^{m}}{(n ! k !)^{l}} a^{n l} b^{k l} \int_{0}^{a} \int_{0}^{b}\left|x^{(n, k)}(s, t)\right| \mathrm{d} s \mathrm{~d} t .
$$

Proof. It is clear that

$$
\begin{aligned}
|x(s, t)| \leq & \frac{1}{(n-1) !(k-1) !} \int_{0}^{s} \int_{0}^{t}(s-\sigma)^{n-1}(t-\tau)^{k-1}\left|x^{(n, k)}(\sigma, \tau)\right| \mathrm{d} \sigma \mathrm{d} \tau \\
& \leq \frac{1}{(n-1) !(k-1) !} s^{n-1} t^{k-1} \int_{0}^{s} \int_{0}^{t}\left|x^{(n, k)}(\sigma, \tau)\right| \mathrm{d} \sigma \mathrm{d} \tau
\end{aligned}
$$

and hence

$$
\begin{gathered}
\int_{0}^{a} \int_{0}^{b}|x(s, t)|^{l}\left|x^{(n, k)}(s, t)\right|^{m} \mathrm{~d} s \mathrm{~d} t \\
\leq \frac{1}{[(n-1) !(k-1) !]^{l}} \int_{0}^{a} \int_{0}^{b} s^{(n-1) l} t^{(k-1) l}\left|x^{(n, k)}(s, t)\right|^{m}\left(\int_{0}^{s} \int_{0}^{t}\left|x^{(n, k)}(s, t)\right| \mathrm{d} s \mathrm{~d} t\right)^{l} \mathrm{~d} s \mathrm{~d} t .
\end{gathered}
$$


Now applying Hölder inequality with indices $\frac{1}{l}$ and $\frac{1}{m}$, we obtain

$$
\begin{gathered}
\int_{0}^{a} \int_{0}^{b}|x(s, t)|^{l}\left|x^{(n, k)}(s, t)\right|^{m} \mathrm{~d} s \mathrm{~d} t \leq \frac{1}{[(n-1) !(k-1) !]^{l}}\left(\int_{0}^{a} \int_{0}^{b} s^{n-1} t^{k-1} \mathrm{~d} s \mathrm{~d} t\right)^{l} \\
\times\left(\int_{0}^{a} \int_{0}^{b}\left|x^{(n, k)}(s, t)\right|\left(\int_{0}^{s} \int_{0}^{t}\left|x^{(n, k)}(\sigma, \tau)\right| d \sigma d \tau\right)^{\frac{l}{m}} \mathrm{~d} s \mathrm{~d} t\right)^{m} \\
=\frac{1}{[(n-1) !(k-1) !]^{l}}\left(\frac{1}{n ! k !}\right)^{l} a^{n l} b^{k l} \\
\times\left(m \int_{0}^{a} \int_{0}^{b} \frac{\partial^{2}}{\partial s \partial t}\left\{\left(\int_{0}^{s} \int_{0}^{t}\left|x^{(n, k)}(\sigma, \tau)\right| d \sigma d \tau\right)^{\frac{l}{m}+1}\right\} \mathrm{d} s \mathrm{~d} t\right)^{m} \\
=\frac{m^{m}}{(n ! k !)^{l}} a^{n l} b^{k l} \int_{0}^{a} \int_{0}^{b}\left|x^{(n, k)}(s, t)\right| \mathrm{d} s \mathrm{~d} t .
\end{gathered}
$$

This completes the proof.

Remark 2.7. Let $x(s, t)$ reduce to $s(t)$ and with suitable modifications. Then (2.16) becomes the following inequality:

$$
\int_{0}^{a}\left|x^{(k)}(t)\right|^{l}\left|x^{(n)}(t)\right|^{m} \mathrm{~d} t \leq \frac{m^{m}}{(n !)^{l}} a^{n l} \int_{0}^{a}\left|x^{n}(t)\right| \mathrm{d} t .
$$

This is an inequality given by Das [28].

Remark 2.8. Following Remark 2.3, for $x(s, t) \in C^{(n-1)}[0, a] \times C^{(k-1)}[0, b]$, $\frac{\partial^{j}}{\partial t} x(s, 0)=0, \frac{\partial^{j}}{\partial t} x(s, 0)=0, \alpha \leq i \leq n-1, \beta \leq j \leq k-1$, and $x^{(n-1, k-1)}(s, t)$ are absolutely continuous, and $\int_{0}^{a} \int_{0}^{b}\left|x^{(n, k)}(s, t)\right| \mathrm{d} s \mathrm{~d} t<\infty$, from (2.16), it is easy to obtain that

$$
\begin{gathered}
\int_{0}^{a} \int_{0}^{b}\left|x^{(\alpha, \beta)}(s, t)\right|^{l} \cdot\left|x^{(n, k)}(s, t)\right|^{m} \mathrm{~d} s \mathrm{~d} t \\
\leq \frac{m^{m}}{[(n-\alpha) !(k-\beta) !]^{l}} a^{l(n-\alpha)} b^{l(k-\beta)} \int_{0}^{a} \int_{0}^{b}\left|x^{(n, k)}(s, t)\right| \mathrm{d} s \mathrm{~d} t .
\end{gathered}
$$

Let $x(s, t)$ reduce to $s(t)$ and with suitable modifications, then (2.16) becomes the following inequality:

$$
\int_{0}^{a}\left|x^{(k)}(t)\right|^{l}\left|x^{(n)}(t)\right|^{m} \mathrm{~d} t \leq \frac{m^{m}}{((n-k) !)^{l}} a^{(n-k) l} \int_{0}^{a}\left|x^{n}(t)\right| \mathrm{d} t, l+m=1 .
$$

This is an inequality given by Das [28].

\section{Acknowledgements}

The authors express their grateful thanks to the referee for his many very valuable suggestions and comments. Research of Chang-Jian Zhao was supported by National Natural Science Foundation of China (10971205). Research of Wing-Sum Cheung was partially supported by a HKU URC grant.

\section{Author details}

${ }^{1}$ Department of Mathematics, China Jiliang University, Hangzhou 310018, PR China ${ }^{2}$ Department of Mathematics, The University of Hong Kong, Pokfulam Road, Hong Kong 


\section{Authors' contributions}

C-JZ and W-SC jointly contributed to the main results Theorems 2.1, 2.2, and 2.3. Both authors read and approved the final manuscript.

\section{Competing interests}

The authors declare that they have no competing interests.

Received: 28 December 2010 Accepted: 17 June 2011 Published: 17 June 2011

\section{References}

1. Opial, Z: Sur une inégalité. Ann Polon Math. 8, 29-32 (1960)

2. Agarwal, RP, Pang, PYH: Opial Inequalities with Applications in Differential and Difference Equations. Kluwer Academic Publishers, Dordrecht (1995)

3. Agarwal, RP, Lakshmikantham, V: Uniqueness and Nonuniqueness Criteria for Ordinary Differential Equations. World Scientific, Singapore. (1993)

4. Bainov, D, Simeonov, P: Integral Inequalities and Applications. Kluwer Academic Publishers, Dordrecht (1992)

5. Li, JD: Opial-type integral inequalities involving several higher order derivatives. J Math Anal Appl. 167, 98-100 (1992). doi:10.1016/0022-247X(92)90238-9

6. Mitrinovič, DS, Pečarić, JE, Fink, AM: Inequalities involving Functions and Their Integrals and Derivatives. Kluwer Academic Publishers, Dordrecht (1991)

7. Cheung, WS: On Opial-type inequalities in two variables. Aequationes Math. 38, 236-244 (1989). doi:10.1007/ BF01840008

8. Cheung, WS: Some new Opial-type inequalities. Mathematika. 37, 136-142 (1990). doi:10.1112/50025579300012869

9. Cheung, WS: Some generalized Opial-type inequalities. J Math Anal Appl. 162, 317-321 (1991). doi:10.1016/0022-247X (91)90152-P

10. Cheung, WS: Opial-type inequalities with $m$ functions in $n$ variables. Mathematika. 39, 319-326 (1992). doi:10.1112/ S0025579300015047

11. Cheung, WS, Zhao, DD, Pečarić, JE: Opial-type inequalities for Differential Operators. Nonlinear Anal. in press (2011)

12. Godunova, EK, Levin, V: On an inequality of Maroni. Mat Zametki. 2, 221-224 (1967)

13. Mitrinovič, DS: Analytic Inequalities. Springer-Verlag, Berlin, New York (1970)

14. Pachpatte, BG: On integral inequalities similar to Opial's inequality. Demonstratio Math. 22, 21-27 (1989)

15. Pachpatte, BG: On integral inequalities similar to Opial's inequality. Demonstratio Math. 22, $21-27$ (1989)

16. Pachpatte, BG: Some inequalities similar to Opial's inequality. Demonstratio Math. 26, 643-647 (1993)

17. Pachpatte, BG: A note on generalized Opial type inequalities. Tamkang J Math. 24, 229-235 (1993)

18. Pečarić, JE: An integral inequality, in Analysis, Geometry, and Groups: A Riemann Legacy Volume in: Srivostava, HM, Rassias, ThM (eds) Part II. pp. 472-478. Hadronic Press, Palm Harbor, Florida (1993)

19. Pečarić, JE, Brnetić, I: Note on generalization of Godunova-Levin-Opial inequality. Demonstratio Math. 30, 545-549 (1997)

20. Pečarić, JE, Brnetić, I: Note on the Generalization of Godunova-Levin-Opial inequality in Several independent Variables. J Math Anal Appl. 215, 274-282 (1997). doi:10.1006/jmaa.1997.5529

21. Rozanova, Gl: Integral inequalities with derivatives and with arbitrary convex functions. Moskov Gos Ped Inst Vcen Zap. 460, 58-65 (1972)

22. Yang, GS: Inequality of Opial-type in two variables. Tamkang J Math. 13, 255-259 (1982)

23. Agarwal, RP: Sharp Opial-type inequalities involving $r$-derivatives and their applications. Tohoku Math J. 47(4):567-593 (1995). doi:10.2748/tmj/1178225462

24. Agarwal, RP, Pang, PYH: Sharp opial-type inequalities in two variables. Appl Anal. 56(3):227-242 (1996). doi:10.1080/ 00036819508840324

25. Alzer, H: An Opial-type inequality involving higher-order derivatives of two functions. Appl Math Letters. 10(4):123-128 (1997). doi:10.1016/50893-9659(97)00071-2

26. Karpuz, B, Kaymakcalan, B, Özkan, UM: Some multi-dimenstonal Opial-type inequalities on time scales. J Math Ineq. 4(2):207-216 (2010)

27. Zhao, CJ, Cheung, WS: Sharp integral inequalities involving high-order partial derivatives. J Ineq Appl. 2008, Article ID $57141710(2008)$

28. Das, KM: An inequality similar to Opial's inequality. Proc Amer Math Soc. 22, 258-261 (1969)

29. Willett, D: The existence-uniqueness theorem for an $n$-th order linear ordinary differential equation. Amer Math Monthly. 75, 174-178 (1968). doi:10.2307/2315901

30. Beesack, PR: On an integral inequality of Z. Opial Trans Amer Math Soc. 104, 470-475 (1962). doi:10.1090/S0002-99471962-0139706-1

31. Agarwal, RP, Thandapani, E: On some new integrodifferential inequalities. Anal sti Univ "Al. I. Cuza" din lasi. 28, 123-126 (1982)

32. Yang, GS: On a certain result of Z. Opial Proc Japan Acad. 42, 78-83 (1966). doi:10.3792/pja/1195522120

doi:10.1186/1029-242X-2011-7

Cite this article as: Zhao and Cheung: On some Opial-type inequalities. Journal of Inequalities and Applications 2011 $2011: 7$. 